\title{
The TELSAM Protein Polymer significantly Improves the Speed of Crystallization of target proteins \\ sara soleimani ${ }^{1}$, Supeshala Sarath Nawarathnage ${ }^{2}$, Parag Gajjar ${ }^{3}$, Braydan Bezzant ${ }^{4}$, Moriah Longhurst ${ }^{5}$, Tobin Smith ${ }^{6}$, Maria Pedroza Romo ${ }^{7}$, Seth Brown ${ }^{8}$, Tzanko Duokov ${ }^{9}$, James Moody ${ }^{10}$, Diana Ramirez ${ }^{11}$ \\ ${ }^{1}$ No affiliation given ${ }^{2}$ No affiliation given, ${ }^{3}$ Brigham Young University, ${ }^{4}$ Brigham Young University, ${ }^{5}$ Brigham Young University, ${ }^{6}$ Brigham Young University, ${ }^{7}$ Brigham Young University, ${ }^{8}$ Brigham Young University, ${ }^{9}$ Macromolecular Crystallography Group, Structural Molecular Biology Resource, SSRL, ${ }^{10}$ No affiliation given, ${ }^{11}$ California State Univesity, Chico sara@chem.byu.edu
}

The development of novel crystallization approaches that require less time, effort, and expense can significantly increase the success rate of target proteins crystallization and accelerate structure determination of many biotechnology and disease-relevant proteins. Specifically, improved crystallization methods will accelerate study of the molecular mechanisms of disease. Polymer forming crystallization chaperones (PFCCs) are a potentially better type of crystallization chaperone. One potential PFCC is the sterile alpha motif domain (SAM) of the human Translocation ETS Leukemia protein. In this study, the effectiveness of TELSAM protein polymers to reliably form well-diffracting crystals of Capillary Morphogenesis Gene 2 (CMG2) is investigated. CMG2 is involved in cancer, where its overexpression is associated with increased tumor grade and poor patient survival. The long-term goal of this research is to develop crystallization methods that could lead to diffraction-quality crystals from greater than $70 \%$ of targeted proteins of interest. A TELSAM-target protein fusion can crystallize more rapidly than the same target protein alone. In this work the results on CMG2 alone, 1TEL-flex-CMG2, 1TEL-flex-CMG2+PGM, 1TELflex-DARPin will be presented. TELSAM accelerates the rate of crystal formation by as much as 27 -fold versus the target protein alone, likely by using avidity to stabilize weak crystal contacts made by the target protein. In addition, TELSAM-target protein fusions can form well-ordered, diffracting crystals using flexible TELSAM-target linkers. The TELSAM polymers themselves need not directly touch one another in the crystal lattice to make crystal lattice. We conclude that TELSAM is a powerful crystallization chaperone warranting future investigation. 